\title{
Computer Simulation of Rolling Textures Evolution of Pure Aluminum with Initial Texture
}

\author{
Zhiyong Chen ${ }^{1,2}$, Xinming Zhang ${ }^{1}$, Chuming Liu ${ }^{1}$, Hongnian Cai ${ }^{2}$ and Fuchi Wang ${ }^{2}$ \\ ${ }^{1}$ College of Materials Science and Engineering, Central South University, Changsha 410083 P.R. China \\ ${ }^{2}$ College of Materials Science and Engineering, Beijing Institute of Technology, Beijing 100081 P.R. China
}

\begin{abstract}
The experimental of rolling texture development in pure aluminum with initial texture was carried out. The rolling texture mainly consists of components B and S while the C component is also observed. The rolling textures evolution was simulated by the Taylor-type models. For the full constraints (FC) Taylor model, the mean method over all the possible solutions was used to solve the ambiguity in the selection of the active slip systems. For the relaxed constraints (RC) lath and pancake models, the ambiguity can be solved effectively by some modified principle, in which the strain compatibility can be fulfilled as much as possible. It has been shown that using the principles proposed in this paper, the rolling texture formation and development of pure aluminum with initial texture could be explained by applying different models under different deformation degree.
\end{abstract}

(Received March 4, 2004; Accepted July 6, 2004)

Keywords: Taylor-type models, rolling deformation, initial texture, computer simulation

\section{Introduction}

It is well known that texture has very important impacts on the properties of materials. Many investigations have been carried out about the texture prediction of polycrystalline materials according to the deformation conditions. Generally, the deformation textures simulation of polycrystalline aggregates may be based on the Sachs ${ }^{1)}$ and the Taylor ${ }^{2)}$ models, as well as some modified models. ${ }^{3-6)}$ Some researches have shown ${ }^{7,8)}$ that the Taylor-type models are closer to experimental results than the Sachs model. However, when the full constraints (FC) Taylor-model is applied to simulate the deformation textures of face-centered-cubic (fcc) metals, a problem arises in that the yield conditions can be fulfilled by more than the necessary 5 slip systems, namely 6 or 8 slip systems. For the relaxed constraints (RC) Taylormodels, this ambiguity in selecting active slip systems still exists while the extent reduced. In the past investigations, some different methods were proposed to solve this problem. ${ }^{9-15)}$ However, most investigation on the prediction of deformation textures were carried out based on a randomly distributed texture and the influence of initial textures did not be considered. As for f.c.c. metals with high stacking fault energy, initial texture has important effects on the evolution of the rolling textures since slip is the main deformation mechanism. Taking the initial texture into account, the evolution of the rolling textures of pure aluminum with initial texture was simulated using Taylor-type models. It has been shown that the simulated results agree with the measured rolling textures by applying different models under different rolling degree.

\section{Experiments and Results}

The specimen of pure aluminum with an initial texture was obtained by properly deformation and heat-treatment. The specimen was rolled to 5 different thicknesses to obtain rolling reductions ranging from $50 \%$ to $95 \%$. Samples for texture investigation were taken at $50 \%, 70 \%, 80 \%, 90 \%$,
95\% reduction. Texture measurements were carried out on a fully automatic and computer-controlled texture goniometer. Four incomplete pole figures $\{111\},\{200\},\{220\}$ and $\{311\}$ were obtained after correction and symmetrization. From these pole figures data the corresponding ODFs (orientation distribution functions) were calculated using the series expansion method according to Bunge. The ghost effect was corrected and the true ODFs were calculated by the method proposed by Lücke. ${ }^{16)}$ Figures 1(a)-(f) are the measured ODFs of the samples for the different reduction.

Figure 1(a) shows that the initial texture in pure aluminum is not randomly distributed, most crystallites aggregate around the cube orientation. The orientation position with the maximum density is not situated at the cube orientation, but at $\left\{15^{\circ}, 0^{\circ}, 0^{\circ}\right\}$. From Figs. 1(b)-(f), the formation and development of a typical pure metal type rolling texture can be observed with increased rolling deformation. Since the mail features of the rolling textures development in pure aluminum can be characterized by plotting of intensities along certain orientation lines called $\alpha$ and $\beta$ fibres, the corresponding skeleton line analysis is shown in Fig. 2. It can be seen that the intensities are distributed inhomogeneously along $\alpha$ and $\beta$ lines for different degrees of rolling. With increased rolling, the density at orientation $\{011\}\langle 111\rangle$ decreases while that at orientation $\{011\}\langle 211\rangle$ increases along $\alpha$ fibre. The density along the whole $\beta$ line increases continuously with deformation. At the high deformation degree, it can been seen from Fig. 1 and Fig. 2 that the rolling texture for pure aluminum with initial texture consists of three main components $C\{211\}\langle 111\rangle, \mathrm{S}\{123\}\langle 634\rangle$ and $\mathrm{B}$ $\{011\}\langle 211\rangle$. Though the density at $\mathrm{C}$ and $\mathrm{S}$ orientations is almost the same, the $\mathrm{S}$ orientation gains the highest volume fraction because of its lower symmetry compared to $\mathrm{C}$ $\{211\}\langle 111\rangle$ and B $\{011\}\langle 211\rangle$ components.

\section{Simulation}

\subsection{Representation of initial orientations}

Figure 1(a) shows that the initial texture in pure aluminum 


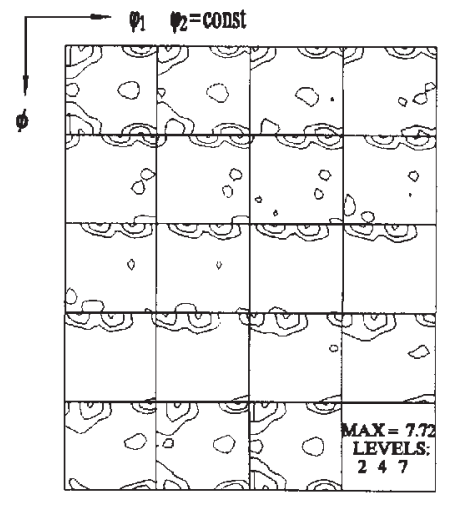

(a) $0 \%$

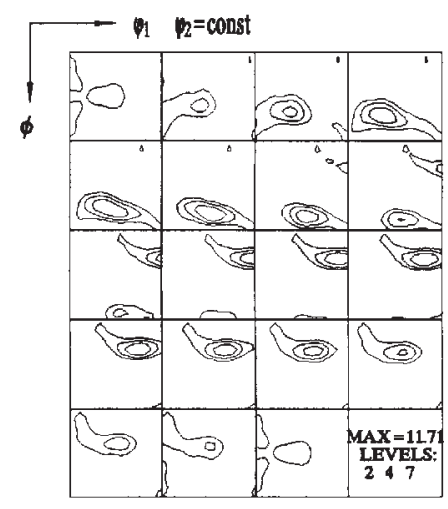

(d) $80 \%$

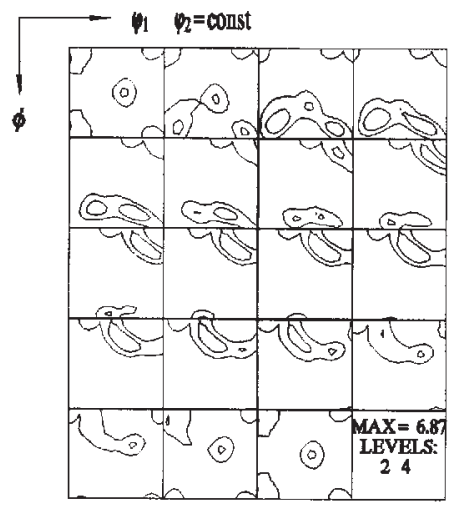

(b) $50 \%$

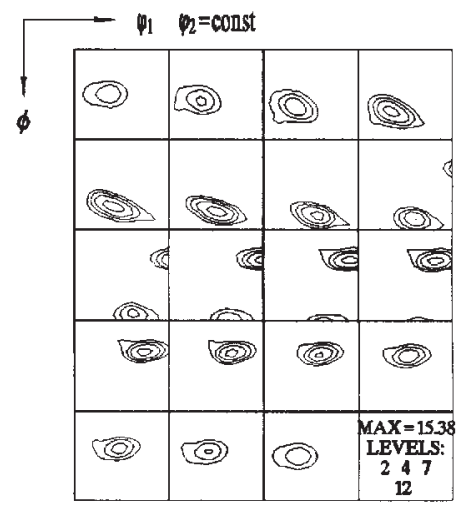

(e) $90 \%$

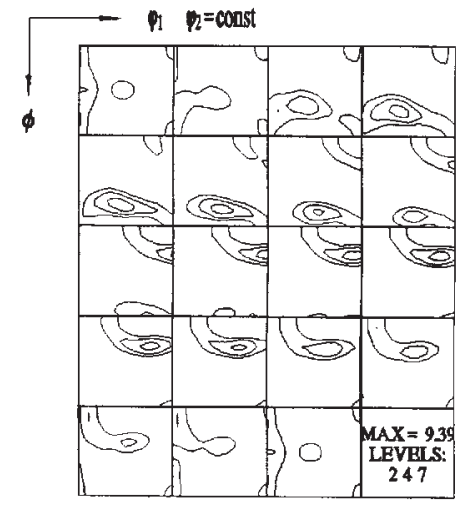

(c) $70 \%$

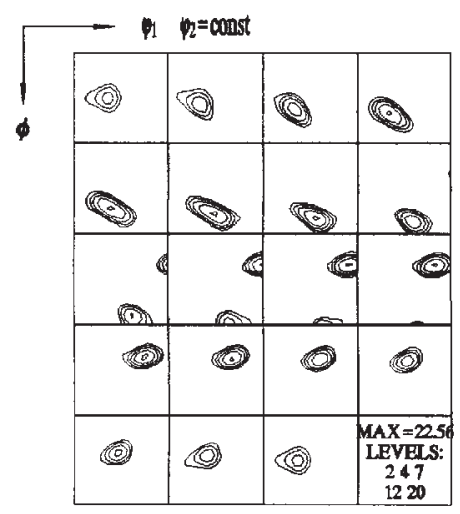

(f) $95 \%$

Fig. 1 Complete experimental ODFs of rolling texture of pure aluminum for different reduction.

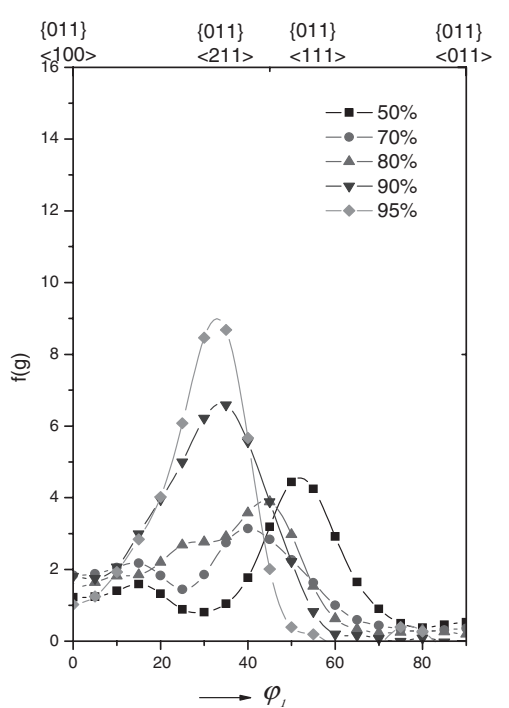

(a) $\alpha$-fibre

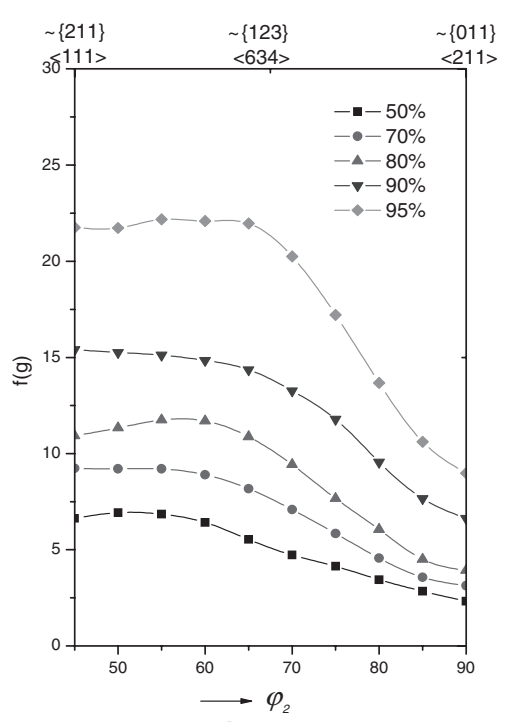

(b) $\beta$ - fibre

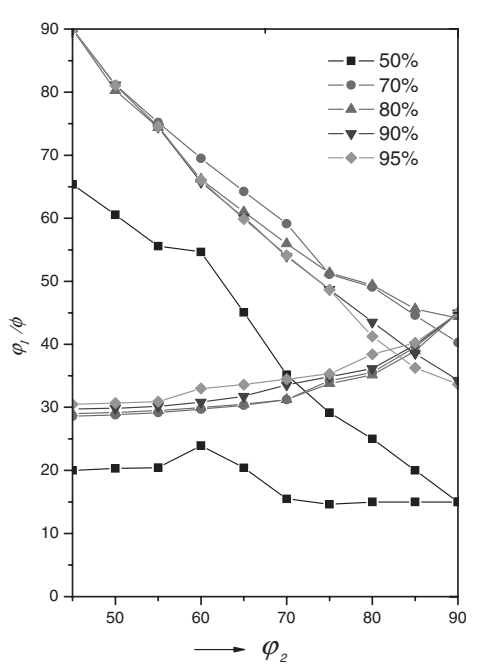

(c) $\beta$ - fibre position

Fig. 2 Orientation line analysis of measured rolling texture.

sample is not randomly distributed, so the influence of initial texture on the evolution of the rolling textures should be considered. Considering the cubic symmetry of the crystal structure and the orthogonal symmetry of the rolling sample, all the orientations of the crystals can be represented by the I subspace in orientation space, that is, the following Euler subspace is sufficient to describe the orientations distribution function of the rolling texture:

$$
\begin{array}{ll}
\text { For } & \varphi_{1} \quad \text { and } \varphi_{2}: \varphi_{1}, \varphi_{2} \in[0, \pi / 2] \\
\text { For } & \phi: \phi \in\left[0, \arctan \left(1 / \cos \varphi_{2}\right)\right], \text { for } \varphi_{2} \leq \pi / 4 \\
& \phi \in\left[0, \arctan \left(1 / \sin \varphi_{2}\right)\right], \quad \text { for } \varphi_{2} \geq \pi / 4
\end{array}
$$


The subspace is divided into 1620 small spaces where the central orientation $g_{i}$ is its characteristic orientation. ${ }^{17)}$ For the sample with initial texture, since the $C_{1}^{\mathrm{uv}}$ coefficients and the true ODF have been known, in order to express the sample with initial texture effectively, the orientation density $f(g)$ of the 1620 discrete orientations has been calculated using the $C_{1}^{\mathrm{uv}}$ coefficient:

$$
f(g)=1+\sum_{l=4}^{23} \sum_{u=1}^{M(l)} \sum_{v=1}^{N(l)} C_{1}^{\mathrm{uv}} \dot{T}_{1}^{\mathrm{uv}}\left(\varphi_{1}, \phi, \varphi_{2}\right)
$$

Then the proper weighting factors have been given to the discrete orientation as its volume fractions according to the orientation density $f(g)$. In this paper, each orientation point was replaced by a Gauss-type component with a half width of $7^{\circ}$. Using the above method, the initial texture of the sample can be converted into a series of individual orientations conveniently and effectively. Furthermore, the divided orientations with certain volume fractions can approximately represent the initial texture of the sample.

\subsection{Simulation method}

The following displacement gradient tensor in the external reference systems is assumed for the simulation of a rolling texture:

$$
E^{\mathrm{s}}=\left(\begin{array}{ccc}
\varepsilon & 0 & 0 \\
0 & 0 & 0 \\
0 & 0 & -\varepsilon
\end{array}\right)
$$

For the Taylor-type models, the macroscopic displacement gradient tensor of the crystals can be expressed by

$$
E^{\mathrm{c}}=\left(\begin{array}{ccc}
\varepsilon & 0 & e_{13} \\
0 & 0 & e_{23} \\
0 & 0 & -\varepsilon
\end{array}\right)
$$

If the $e_{13}$ and $e_{23}$ are assumed to be zero in advance, it is called full constraints (FC) model. If the $e_{13}$ is assumed to be not zero and $e_{23}$ is zero, it is called relaxed constraints (RC) lath model. If the $e_{23}$ is assumed to be not zero and $e_{13}$ is zero, it is called $\mathrm{S}$ model. If the $e_{13}$ and $e_{23}$ are assumed to be not zero, it is called RC pancake model.

According to the Taylor minimum internal principle:

$$
W=\sum_{\mathrm{s}} \tau_{\mathrm{s}}\left|\gamma^{\mathrm{s}}\right|=\min
$$

It can be found that there are several combinations of the active slip systems that can fulfill the eq. (5), so the ambiguity problem of selecting the active slip systems arises. If the selected slip systems are different, the rotation change of the crystal orientation is different too. So in the case that there is a multiplicity in selecting active slip systems, the group which is activated should be determined in order to obtain the crystallite orientation after deformation. For FC model, the average method over all possible solutions was used to solve the ambiguity in this paper. For the different RC models, a modified method was proposed in order to ensure the stain compatibility to the maximum degree. In order to determine the active slip systems, the minimum shear principle has been introduced:

$$
\left|e_{13}\right|+\left|e_{23}\right|=\min
$$

The above principle can solve the ambiguity problem effectively. Although the shear deformation of crystallites is undetermined along the rolling direction or/and transverse direction in advance, the active slip systems can be determined according to the principle and the strain compatibility can be satisfied as much as possible. The different combinations of shear component can be applied for different $\mathrm{RC}$ models, that is, $\left|e_{13}\right|=\min$ is suggested for the lath model and $\left|e_{23}\right|=\min$ for the $\mathrm{S}$ model and $\left|e_{13}\right|+\left|e_{23}\right|=$ min for the pancake model. Using the above principle, the ambiguity problem of the slip systems selection can be solved effectively.

Once the active slip systems have been determined, the orientation change can be calculated from the shear amounts on the corresponding slip systems. The deformation steps were chosen as 0.01 in the simulation, the crystal orientations after a large deformation could be predicted by means of a number of successive small deformation steps. The sample orthogonal symmetry and the crystal cubic symmetry were considered when the corresponding true ODFs were calculated.

\section{Results and Discussion}

Using the Taylor-type models, the development of rolling textures of pure aluminum with initial texture has been simulated. It has been found that the ambiguity extent of the selection of the active slip systems by the FC model is much greater than that by the RC models. There are $4 \sim 12$ groups of active slip systems combinations generally for the FC model while there are 1 6 groups usually for RC lath and S model. The ambiguity extent is the lowest for RC pancake model. Using the principle proposed in this paper, the ambiguity problem in selecting active slip systems can be solved effectively and which group of slip systems can be activated may be decided. Therefore, the change of the crystal orientation can be calculated. For simplicity, only $\alpha$ and $\beta$ fibres and the orientation position of $\beta$ fibre are given, as shown in Fig. 3 to Fig. 5.

From Fig. 2 and Fig. 3, it can be seen that the rolling texture of pure aluminum with initial texture are principally accumulated around $\beta$-fibre in orientation space, which mainly consist of $\mathrm{S}$ and $\mathrm{C}$ components. Some crystals are located at the $\mathrm{B}$ orientation. There are some differences between the measured and the calculated density values along the skeleton lines. It is clearly that the simulated texture is much sharper than the measured using the Taylor-type models while the orientation density predicted by the lath or pancake model is lower than that by the FC model. For the lath model, there is an abrupt point along the $\beta$-fibre when the initial texture is considered. The position of abrupt point is shifted from $\varphi_{2}=70^{\circ}$ to $\mathrm{S}$ orientation. This case can not happen in the simulation if the initial orientations are randomly distributed. For the pancake model, it can be concluded from Fig. 5 that there is no crystal aggregates around $\beta$-fibre when $\varphi_{2}>60^{\circ}$ if the deformation degree is $50 \%$ and $70 \%$. There is much more difference between the 


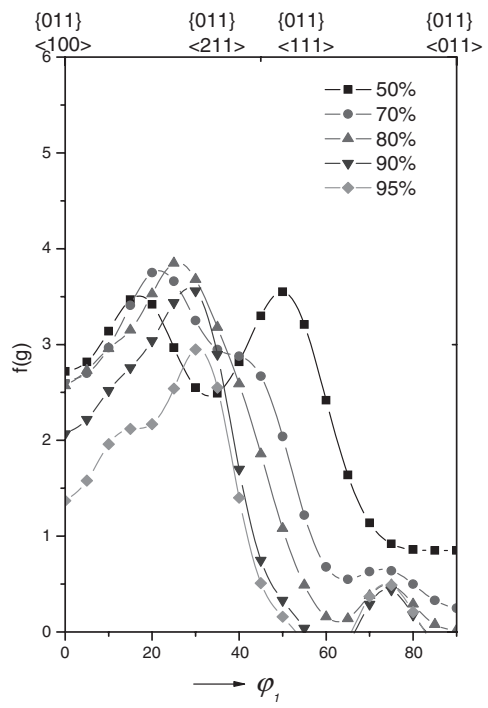

(a) $\alpha$-fibre

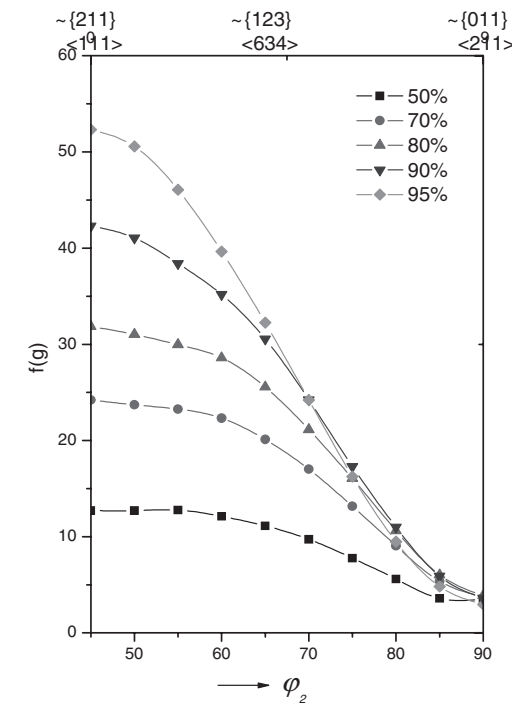

(b) $\beta$-fibre

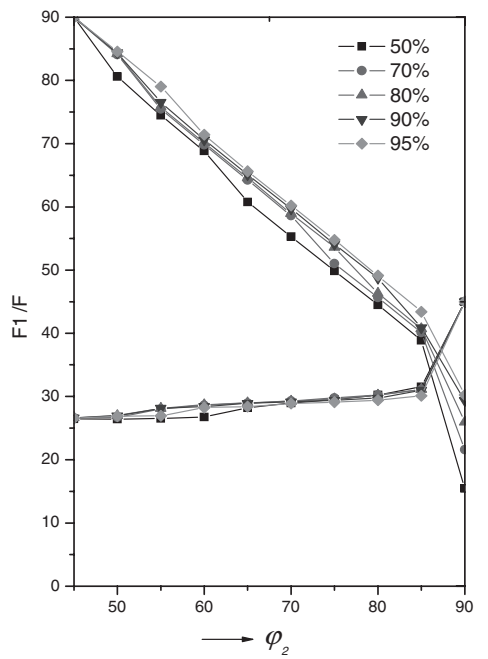

(c) $\beta$-fibre position

Fig. 3 Orientation line analysis of simulated rolling texture by FC model.

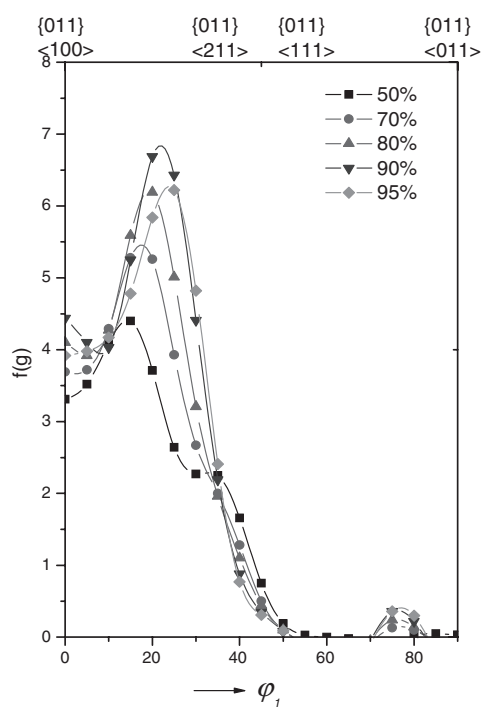

(a) $\alpha$-fibre

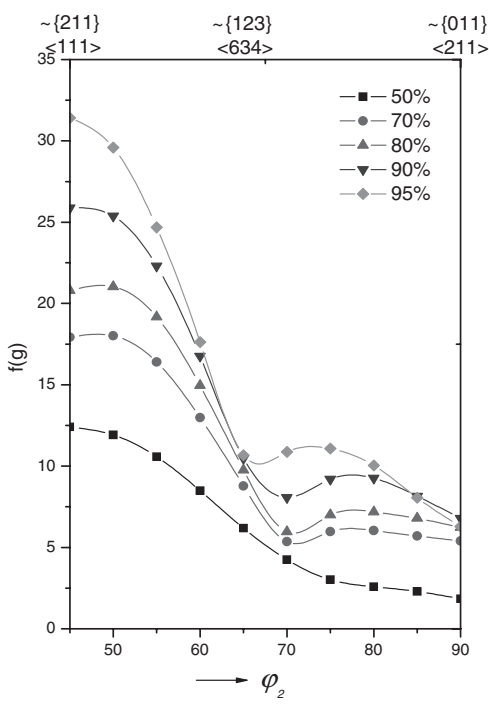

(b) $\beta$-fibre

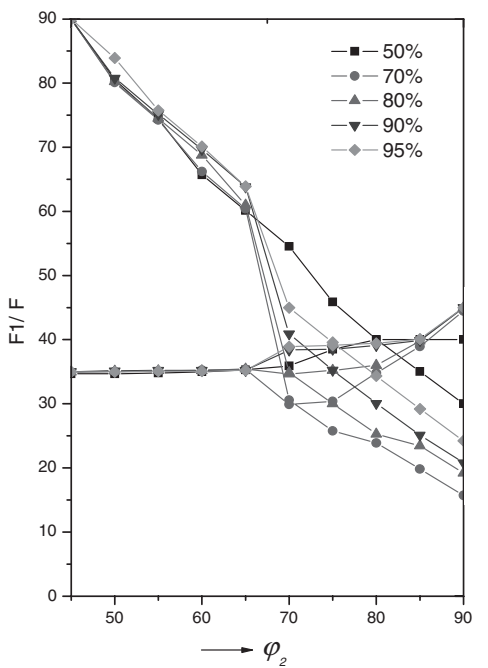

(c) $\beta$-fibre position

Fig. 4 Orientation line analysis of simulated rolling texture by RC lath model.

measured results and the calculated by the pancake model. Comparing the measured results to the simulated by different models in detail, it can be found that the FC model is closer to the fact than the other models at the 50\% rolling deformation degree while there are some differences between the simulated and experimental results. However, all of these models can not explain the evolution of rolling texture of pure aluminum with initial texture well when the different models were used respectively. In fact, when a crystallite in polycrystalline aggregate was deformed, for the same orientation, since the orientations of adjacent grains may be different, correspondingly, the stress and stain states that it experiences may be different.

Hence, for different crystallites with same orientation, they may be deformed in different ways. At the high rolling deformation, the lath and pancake and $\mathrm{S}$ models should be closer the fact while the FC model should be impossible. So we can assume that 40 percent of the crystallites was deformed according to the lath model, 40 percent of the crystallites was deformed according to the pancake mode, 20 percent according to $\mathrm{S}$ model. Taking account of deformation degree, it may be realistic and can occur during rolling. The results of skeleton line are also shown in Fig. 6 for the different reduction. The results showed that the simulated orientation density values by the mixed model are lower than those by other models. It is obviously that the simulated result by using the mixed model is closer to the measured than the simulated results by different models respectively at the deformation degree which is higher $50 \%$. The results predicted by different models for $90 \%$ reduction in Fig. 7 have also demonstrated this conclusion. However, the mixed model can not explain the formation and the development of 


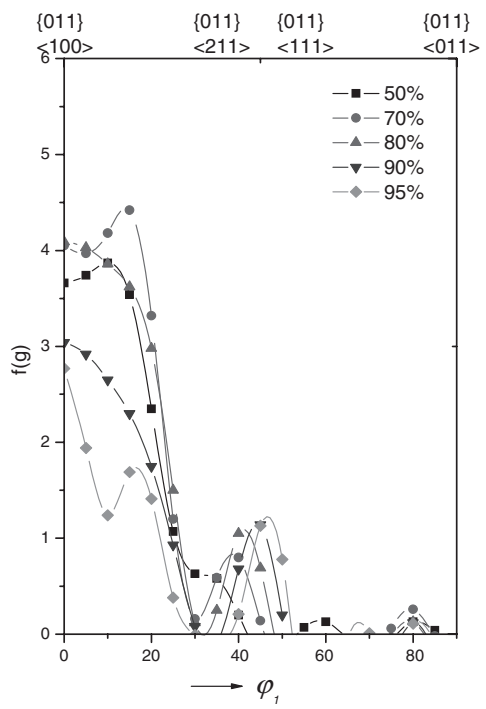

(a) $\alpha$-fibre

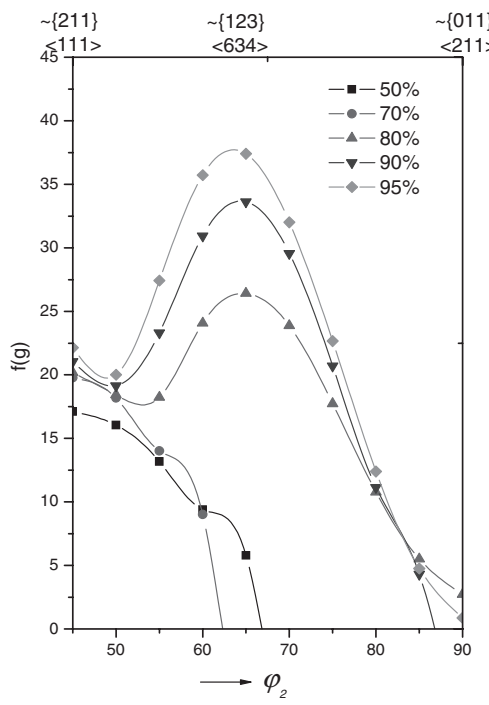

(b) $\beta$-fibre

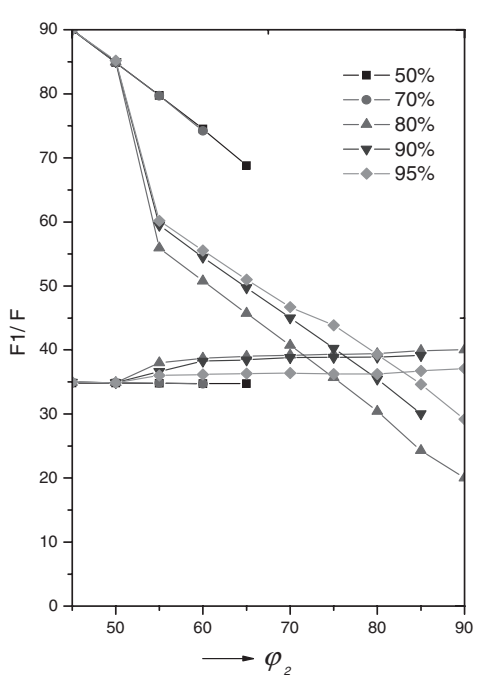

(c) $\beta$-fibre position

Fig. 5 Orientation line analysis of simulated rolling texture by RC pancake model.

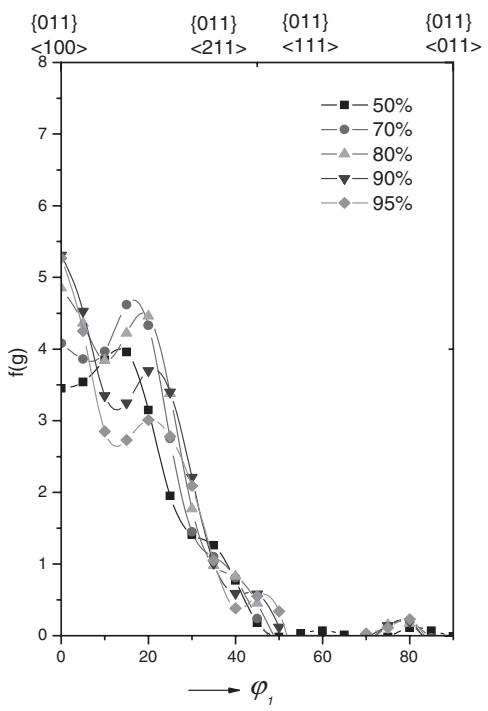

(a) $\alpha$-fibre

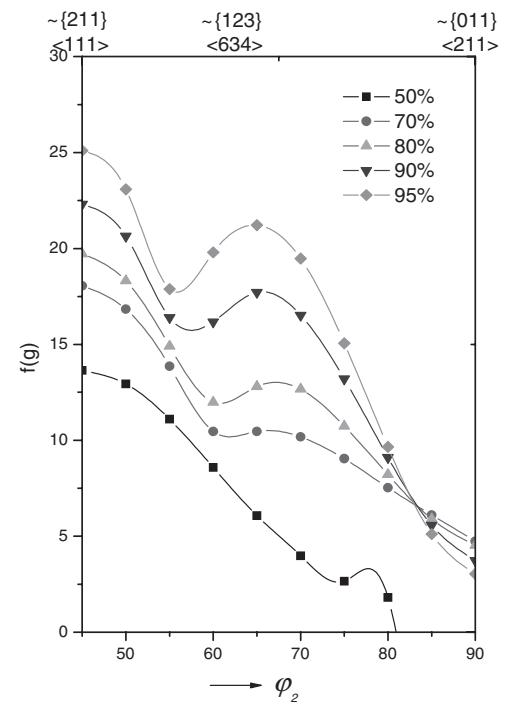

(b) $\beta$ - fibre

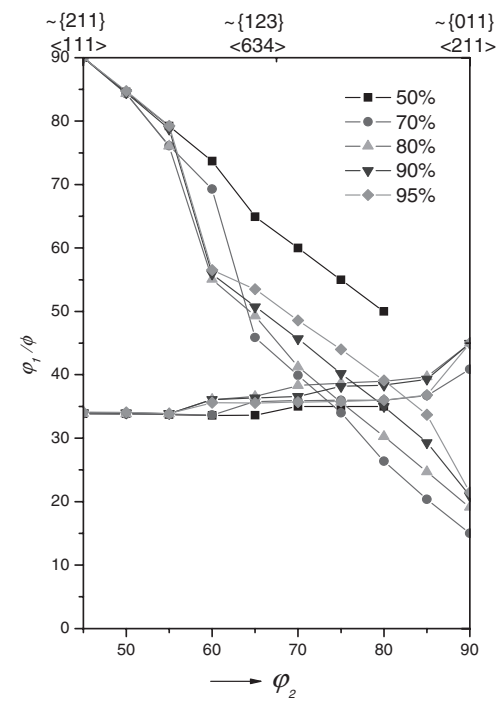

(c) $\beta$-fibre position

Fig. 6 Orientation line analysis of simulated rolling texture by mixed model.

B texture very well. The results show in Fig. 7 that the simulated position of $\mathrm{B}$ orientation is different from the measured. In fact, in order to improve the simulation results, it is necessary to take into account stress equilibrium, strain compatibility, the shape changes of grains, the inhomogeneity of the macroscopic deformation and the microscopic grain deformation, which can be solved by FEM analysis method. ${ }^{18,19)}$ However, it can be seen that the rolling texture evolution of pure aluminum with initial texture could be explained by applying different models under different deformation degree.

\section{Conclusion}

The rolling texture in pure aluminum with nearly cube texture mainly consists of components $\mathrm{B}$ and $\mathrm{S}$ while the orientation density of $\mathrm{C}$ component is the lowest. The Taylortype models were used to simulate the evolution of the rolling textures of pure aluminum. For FC model, the average method was used to solve the ambiguity. For RC models, the ambiguity can be solved effectively by the minimum shear principle in order that the strain compatibility was satisfied to the maximum extent. It has been shown that at the $50 \%$ deformation degree the results by FC model are closer the measured than those by other models. At the higher rolling degree, the simulated results by the mixed models show a better agreement with the experiment compared with other models. 


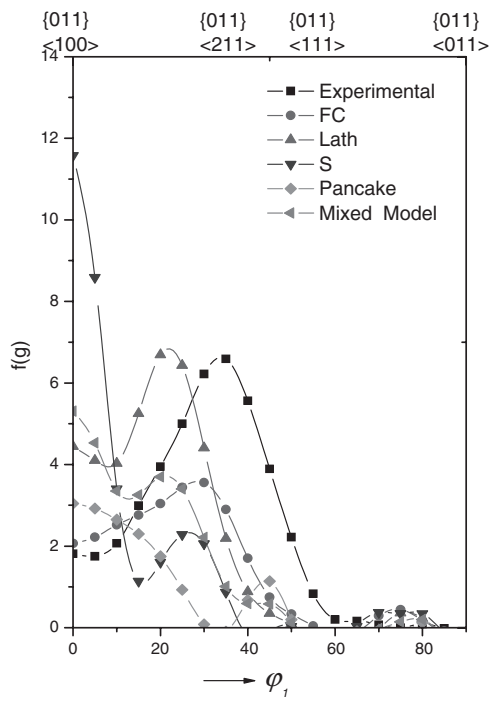

(a) $\alpha$ - fibre

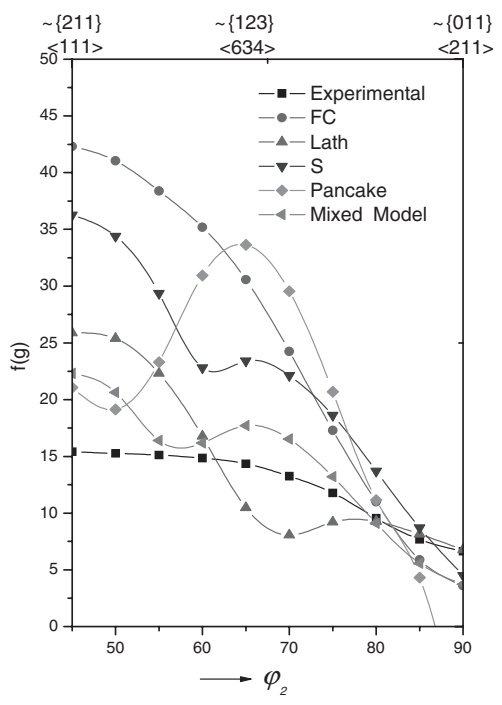

(b) $\beta$ - fibre

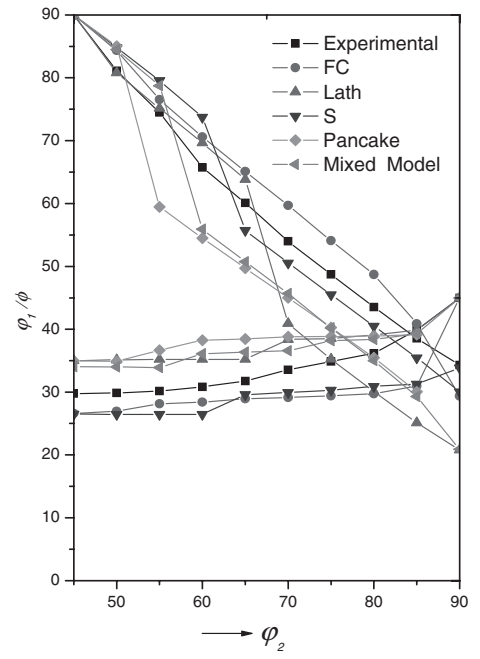

(c) $\beta$ - fibre position

Fig. 7 Orientation line analysis of simulated rolling texture by different models for $90 \%$ reduction.

\section{Acknowledgements}

The authors would like to thank the National Natural Science Foundation Council for the financial support (Projects 50301016 and 50231030) and the Institut für Metallkunde und Metallphysik an der TH-Aachen, Germany for sample's measurements.

\section{REFERENCES}

1) G. Sachs: Z. Ver. Deut. Ing. 72 (1928) 734-736.

2) G. I. Taylor: J. Inst. Metals. 62 (1938) 307-324.

3) H. Honneff and H. Mecking: Proc. 5th Int. Conf. on textures of Materials, ed. by G. Gottstein and K. Lücke, (Berlin: Springer-Verlag, Berlin, 1978) 262-275.

4) P. Van Houtte: Proc. 6th Int. Conf. on textures of Materials, ed. by S. Nagashima, (Iron and Steel Inst. of Japan, Tokyo, 1981) 428-436.

5) U. F. Kocks and H. Chandra: Acta. Metall. 30 (1982) 695-709.

6) R. Fortunier and J. H. Driver: Acta. Metall. 35 (1987) 509-517.
7) U. F. Kocks: Metall. Trans. 1 (1970) 1121-1143.

8) J. Hirsch and K. Lücke: Acta. Metall. 36 (1988) 2883-2904.

9) H.-J. Bunge and T. Leffers: Scr. Metall. 5 (1971) 143-149.

10) H.-J. Bunge: Kristall Techn. 9 (1974) 1051-1071.

11) M. Renouard and M. Wintenberger: C. R. A. S. Seris. B 283 (1976) 237-240.

12) M. Renouard and M. Wintenberger: C. R. A. S. Seris. B 385 (1981) 385-388.

13) H. Mecking: Proc. 6th Int. Conf. on textures of Materials, ed. by S. Nagashima, (Iron and Steel Inst. of Japan, Tokyo, 1981) 53-66.

14) L. Dezillie, P. Van Houtte and E. Aernoudt: Proc. 8th Int. Conf. on textures of Materials, ed. by J. S. Kallend and G. Gottstein, (Warrenale TMS, PA, 1988) 357-368.

15) R. J. Asaro and A. Needleman: Acta. Metall. 33 (1985) 923-953.

16) K. Lücke, J. Pospiech, K. H. Virnich and J. Jura: Acta. Metall. 29 (1981) 167-185.

17) Z. Y. Chen, X. M. Zhang, Z. P. Zhou, S. Y. Li and Y. Yang: Trans. Nonferrous. Met. Soc. China. 9 (1999) 307-311.

18) O. Engler, M.-Y. Huh and C. N. Tomé: Metall. Mater. Trans. A 31A (2000) 2299-2315.

19) F. Pérocheau and J. H. Drive: Int. J. Plast. 16 (2000) 73-89. 